AperTO - Archivio Istituzionale Open Access dell'Università di Torino

Chemical genetics approach to identify new small molecule modulators of cell growth by phenotypic screening of Saccharomyces cerevisiae strains with a library of morpholine-derived compounds

This is the author's manuscript

Original Citation:

Availability:

This version is available http://hdl.handle.net/2318/1715249

since 2019-11-09T22:27:14Z

Published version:

DOI:10.1039/c0ob00357c

Terms of use:

Open Access

Anyone can freely access the full text of works made available as "Open Access". Works made available under a Creative Commons license can be used according to the terms and conditions of said license. Use of all other works requires consent of the right holder (author or publisher) if not exempted from copyright protection by the applicable law. 


\section{Chemical genetics approach to identify new small molecule modulators of cell growth by phenotypic screening of Saccharomyces cerevisiae strains with a library of morpholine-derived compounds}

Andrea Trabocchi, ${ }^{\star a}$ Irene Stefanini, ${ }^{b}$ Manfredi Morvillo, ${ }^{a}$ Leonardo Ciofi, ${ }^{a}$ Duccio Cavalieri ${ }^{\star b}$

5 and Antonio Guarna ${ }^{a}$

aDepartment of Chemistry "Ugo Schiff", University of Florence, Polo Scientifico e Tecnologico, Via della Lastruccia 13, I-50019, Sesto F.no, Florence, Italy. E-mail: andrea.trabocchi@unifi.it; Fax: +39055 4573531;Tel: +39055 4573507

bDepartment of Pharmacology "Mario Aiazzi Mancini", University of Florence, Viale Pieraccini

10 6, 50139, Florence, Italy. E-mail: duccio.cavalieri@unifi.it; Fax: +39 055 4271280; Tel: +39 0554271327

\section{Abstract}

A chemical genetics approach has been applied in the screening of yeast deletants strains with a pool of morpholine-derived compounds in order to identify candidate small molecules

15 able to produce phenotypic effects on yeast cells. The analysis of the effects of structurally diverse molecules towards cell growth rate in both exponential and stationary phases provides a tool to select candidate compounds for subsequent assays to identify new chemical entities as chemical probes for drug discovery.

\section{Introduction}

20 A central challenge in chemical genetics is the mapping of "biological-activity space", which involves analysing both protein binding and phenotypic responses with respect to small molecules. ${ }^{1} \mathrm{~A}$ number of high-throughput phenotypic assays have been developed, including assays that measure cell viability or proliferation. ${ }^{2}$ After collecting a large amount of data on the ability of the members of a chemical library to bind to a set of proteins and affect a set of

25 phenotypes, the data can be analysed to determine the relationship between chemical structure and biological activity. Each compound can be assigned a vector that describes the 
quantitative level of binding to each protein, and the quantitative effect this has on each phenotype. Saccharomyces cerevisiae is particularly suited for the high-throughput screening of new chemical entities on whole cell systems due to key features connected to easy

30 manipulation and the versatility of its genome. ${ }^{3}$ The life cycle is particularly rapid,as it divides in about two hours, it is possible to manipulate both haploid and diploid yeast systems. Moreover, it shows high degree of conservation with human cells concerning main biological processes. Yeast cells can be used first of all to identify novel compounds showing an inhibitory effect on growth. Afterwards, functional information about the mode-of-action of

35 selected compounds can be obtained from scoring 5,000 viable yeast haploid deletion mutant strains for hypersensitivity or hyper-resistance to each specific drug, thus identifying pathways that influence cellular response to the toxic compound. ${ }^{4}$ We recently turned our attention to morpholine-containing molecules, as the relevance of such heterocycle in medicinal chemistry is remarkable, being present in several bioactive molecules, such as 40 TACE (TNF-a converting enzyme), 5 MMP (matrix metalloproteinase), and TNF (tumour necrosis factor) inhibitors. 6 Accordingly, we reported on the diversity-oriented synthesis of morpholine scaffolds taking advantage of a two steps process involving the combination of amino acid derivatives as building blocks. ${ }^{7}$ Also, the conformational analysis of peptides containing morpholine-3-carboxylic acid (Mor) suggested this molecule to act as an 45 unexplored proline surrogate. ${ }^{8}$ We applied the reactivity of some morpholine acetals to give access to a library of morpholine-derived molecules, some of which also contained the 2,5 diketopiperazine nucleus, which in turn is considered a privileged scaffold in medicinal chemistry owing to the wide number of bioactive natural products and drugs containing such chemical entity. ${ }^{9}$ The library of new chemical entities was successively applied for the 50 systematic exploration of yeast deletants, so as to classify deletants as a function of bioactive 
molecules. Accordingly, a scatter diagram plotting the effect of the molecules towards yeast cell growth in both the exponential and stationary phases was assessed as a criterion for hit selection. Further experiments allowed for subsequent selection of candidate compounds as chemical probes to be applied in Systems Biology investigations of cell cycle mechanisms.

\section{Results and discussion}

Chemistry Scaffolds I-III were considered for library generation. Scaffold I was obtained from the combination of di methoxy acetaldehyde $\mathrm{A}$ and serine or L-threonine derivatives corresponding to building blocks $\mathrm{B}$ with $\mathrm{R} 1 \mathrm{H}$ or $\mathrm{CH} 3$, respectively (Scheme 1 ). ${ }^{10}$ Scaffold II was obtained as a single stereoisomer in two steps from phenacylbromide $\mathrm{C}$ and threonine

60 derivative $\mathrm{B}$ consisting of a coupling in $\mathrm{N}$-methylpyrrolidone, followed by cyclization in $\mathrm{HCl} / \mathrm{MeOH}$ solution (see ESI data). Scaffold III embedding a bicyclic lactone was obtained from aminoacetaldehyde dimethylacetal $D$ and protected methyl threonate derivative $E$, as reported.7 Starting from scaffolds I-II, a library of compounds embed-ding 2,5diketopiperazine, 2-oxopiperazine, morpholine and 1,4-dihydro-oxazine heterocycles was

65 generated (see Scheme 2 and Table S1 in ESI data for the complete Chart of compounds).

Compounds 4 and $6-10$ were obtained from a two-steps process consisting of a coupling with Fmoc-aa-Cl, followed by Fmoc deprotection with concomitant formation of the 2,5diketopiperazine ring. Library members $11-26$ were obtained by a three-steps one-pot process consisting of 1) coupling of Fmoc-aa- $\mathrm{Cl}, 2)$ 1,4-dihydro-oxazine formation through

70 acid-mediated double bond formation, 3) 2,5-diketopiperazine ring formation by treatment with $30 \%$ piperidine as the Fmoc-deprotecting agent. The stepwise process consisting of an acidbase workup after amino acid coupling allowed for the achievement of the bicyclic structure of compounds 27-35 embedding 2-oxo-piperazine and oxazolidine rings, as evinced by crystallographic data obtained for compound 34 (Fig. 1). The absence of 2,6-lutidine in the 
75 mixture,and the use of catalytic quantities of p-TSA in the second step were found beneficial for the intermediate oxonium species to undergo an attack by the urethane nitrogen atom, followed by a rearrangement towards the new bicyclic species. Finally, compound5was considered in the library to assess the effects of a monocyclic intermediate with respect to parent bicyclic scaffolds. Bicyclic lactone III was employed for the construction of bicyclic

80 diketopiperazines $36-42$ by coupling with bromoacetyl bromide(scaffold IV) or Fmoc-L-Pro-Cl (scaffold V), and subsequent cyclization by treatment with selected primary amines or $30 \%$ piperidine as the Fmoc-deprotection agent (Scheme 3 and TableS1 in ESI data). The hydroxymethyl group was successively processed to access further chemical diversity. Esterification of compounds $43-44$, coming from scaffold $V$ and IV respectively, with myristic

85 acid allowed for the generation of lipidated scaffolds to envisage potential interactions across the cell membrane. Also, acid-catalyzed processes were applied to myristoyl-L-prolinederived compound 43 to obtain the corresponding 1,4-dihydro-oxazine derivative 45 , and to 41-42 possessing the free hydroxy group to obtain the corresponding polycyclic scaffolds 47 and 46 , respectively.

\section{Biology}

The effects of 48 compounds were initially tested at $0.3 \mathrm{mM}$ concentration on the BY4742 wild-type strain (Fig. 2a). Compounds responsible for the variation in cell generation time, which is the time interval required for a yeast cell to divide, or of the O.D. 650 value of the stationary phase (O.D.st), or both, were selected for further characterization. Most of the

95 molecules induced higher fitness on the wild-type strain, as shown by higher O.D.st and lower GenT values, some of which inducing a more intense effect, e.g. 11, 13, 27, and 28. Only two molecules,5and44, induced a decrease in the yeast fitness, assumed from both an O.D.st decrease and a GenT increase. Other molecules, such as6,45and47, induced an O.D. st and 
GenT increase. In this case the increase of the O.D.st can be ascribed to an increase of the

100 cellular volume, rather than to an increase in cell concentration, which should be associated to a lower GenT. The screening on the wild-type strain allowed for the selection of 21 molecules inducing an O.D.st and/or GenT variation in absolute value higher than $10 \%$ or others interesting effects (e.g. decrease of the yeast fitness). Some structural determinants could be drawn for this first run of screening (see Table S1 in ESI file). Activity of compound 3

105 with respect to 2 highlighted the importance of the phenyl substituent at position 6 of the morpholine ring. The role of such substituent was also evident by comparing L-leucinederived hit compound11with respect to the correspondingscaffold15lacking of the phenyl substituent. Remarkable activity of 5 with respect to parent bicyclic compound26emphasized the importance of the 1,4-dihydro-oxazine ring with respect to the 2,5-diketopiperazine moiety,

110 and also the influential role of the bulky hydrophobic Fmoc group. The effect of 42 with respect to 36-40 showed the role of the p-methoxybenzyl group in the pool of com-pounds originated from scaffold IV. Activity of compound44alsoput evidence on the role of lipidated derivatives. Such compounds were thus tested for effects on the BY4742 $\Delta$ erg6, BY4742 $\Delta$ snq2 and BY4742 $\Delta$ pdr3 deletion strains bearing deficiencies in genes involved in 115 cell wall and MDR (Multi Drug Resistance) (Fig. 2B, 2c, and 2d). The use of a pool of strains bearing deletions of genes involved in membrane assembly or function enables the identification of effects that could be concealed by membrane permeation incapability of the molecule. Moreover it allows for the identification of pathways that influence the cellular response to tested compounds (if the deleted gene is the gene coding the compound target, 120 the compound stops inducing the effects showed on the wild-type strain). Erg6p is a methyl transferase, having a downstream role in the ergosterol biosynthesis with respect to one of the morpholine target (Erg24p), and upstream with respect to the other morpholine target 
(Erg2p); Snq2p is drug-efflux pump ABC (ATP-Binding Cassette) transporter conferring resistance to drugs and oxygen radicals; $\mathrm{Pdr} 3$ is a transcriptional co-activator of genes 125 encoding $A B C$ transporters. Compound 3 induced dramatic effects on GenT of BY4742 $\Delta$ snq2 and BY4742 $\Delta p d r 3$ (102.6\% and 157.6\%, respectively), with a proportional O.D.st increase (3.4\% and 9.2\%, respectively), whereas it induced an opposite effect on the BY4742Derg6strain (Gent $-23.7 \%$, O.D.st $-6.1 \%$ ). These results allowed us to hypothesize that3targets the ergosterol biosynthesis pathway downstream Erg6p. Much more intense was

130 the effect of molecule 5 on GenT of the deletant strain BY4742 $\Delta$ erg6 (921.5\%). Interestingly, several molecules showed opposite effects on deletant strains with respect to the wild-type strain. Specifically, molecules 3, 6 and 35, inducing an O.D.st decrease in the wild-type strain, induced the same, though weaker, effect on BY4742 $\Delta$ erg6 strain, and an opposite effect on BY4742 $\Delta p d r 3$ and BY4742 $\Delta s n q 2$ strains, showing that the activity of the molecule is affected

135 by cell MDR (Multi Drug Resistance) systems. Compound 42, inducing an O.D. st decrease in

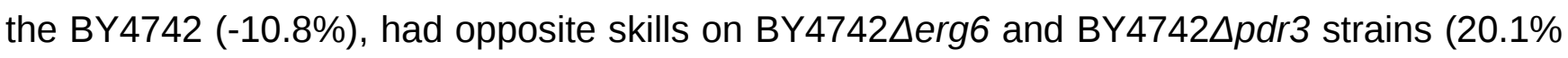
and $4.2 \%$, respectively), indicating a possible transcriptional target. Molecule 5 induced an O.D.st decrease on wild-type, $\Delta$ erg6 and $\Delta p d r 3$ strains $(-28.1 \%,-10.5 \%$ and $-8.5 \%$, respectively), but induced an increase on the $\Delta s n q 2$ strain (5.8\%). Compound 29 induced an

140 O.D.st increase on every deletant strain, differently from the effect on the wild-type strain. The myristoylated compound 44 induced a O.D.st decrease in all the tested strains, with the greatest effect on the BY4742 $\Delta p d r 3$ strain. The eight molecules $(3,5,6,28,29,35,42$, and 44) inducing O.D.st and/or GenT variations higher than $10 \%$ on at least two of the deletant strains were tested for mitochondrial membrane potential activation (Fig. 3) and peroxisomal

145 proliferation (Fig. 4). Mitochondrial membrane activation and peroxisomal proliferation are indicators of the metabolic state of the cell,disclosing respiratory and fatty acid metabolism, 
respectively. The variation in mitochondrial membrane potential is also caused by ion-channel permeability and drug binding. ${ }^{11}$ Peroxisomes are organelles containing enzymes for the boxidation of fatty acids, can be involved in inactivation of toxic substrates $\left(\mathrm{H}_{2} \mathrm{O}_{2}\right.$-based

150 respiration), in the synthesis of ether phospholipids (in mammals) and in the breakdown of purines and amino acids. ${ }^{12}$ Moreover, the peroxisome function and assembly are controlled by ABC proteins. ${ }^{13}$ The mitochondrial activation skill of each molecule was assessed in various metabolic conditions, growing cells in YP supplemented with different carbon sources: glucose and galactose (both fermentable and non fermentable carbon source), glycerol (non

155 fermentable) and oleate (fatty acid, b-oxidable by peroxisomes). All the selected molecules induced lower percentage of mitochondrial activation with respect to the control treatment (with DMSO), in particular the 3 molecule induced the lowest mitochondrial activation in all the media assayed (Fig. 3). The only molecule inducing an increase in mitochondrial activation was42in YPGal. The peroxisomal proliferation was unaltered in every treatment but in the 160 treatment with3and5molecules, in which it was decreased in a statistically significant level. Interestingly,the molecule3induced an apparent wall modification on the strain BY4742 $\operatorname{erg} 6$, as observable by decreased Calcofluor White staining, confirming the hypothesis that this molecule target is the ergosterol biosynthesis pathway (Fig. 5). Our results indicate that the molecules 3 and 5 show bright activities. Compound3, inducing the lowest peroxisomal 165 proliferation and a growth increase in $\Delta s n q 2$ and $\Delta p d r 3$ strains, is a promising powerful instrument to gain further insight into the drug resistance mechanism and acquisition in mammal and yeast cells. On the other hand, compound5, showing killer activity only toward wild-type and $\Delta$ erg6 strains, may be further explored as a suitable chemical probe for assessing the mechanisms underlying the multidrug resistance of pathogenic cells with 170 respect to normal cells. The likely function of molecule 5 on the cells is a starvation induction, 
170 induced by respiration and b-oxidation decrease (observable by the mitochondrial activity and peroxisomal proliferation reduction).

\section{Conclusions}

The process of generating libraries of new chemical entities carrier of chemical diversity, and the screening towards suitable living systems such as Saccharomyces cerevisiae through a

175 chemical genetics approach is a promising and powerful tool to identify candidate small molecules for drug discovery purposes. Accordingly, the graphical inspection of the effects of structurally diverse molecules towards cell growth rate in both exponential and stationary phases through scatter plots provides a rapid and efficient tool to evaluate the effects of library members with respect to different yeast deletant strains, thus enabling the

180 classification of phenotype responses as a function of the chemotype, and to provide new chemical tools for Systems Biology.

\section{Experimental}

\section{General procedure A for the synthesis of compounds 4,6-10 from scaffold I}

Morpholine acetal I (1 eq) was dissolved in toluene(10 $\mathrm{mL} \mathrm{mmol}-1)$, then 2,6-lutidine (2 eq)

185 and $\mathrm{Fmoc}-\mathrm{aa}-\mathrm{Cl}(1 \mathrm{eq})$ were sequentially added. The reaction mixture was brought to60 $\mathrm{C}$ and stirred for $2 \mathrm{~h}$. The reaction mixture was brought back at r.t. and $30 \%$ diethylamine solution in $\mathrm{CH} 3 \mathrm{CN}$ (5 mL mmol-1) was added. The reaction mixture was stirred for $2 \mathrm{~h}$ at r.t., then diluted with EtOAc (40 mL mmol-1), washed with 5\% NaHCO3and brine. The organic layers were dried over anhydrous $\mathrm{Na2SO}$, filtered and evaporated. A dark brown oil was

190 obtained and purified by flash chromatograhy (EtOAc-petroleum ether $2: 1$ ), giving the title2,5-diketopiperazine compounds. See ESI file for characterization data.

General procedure B for the synthesis of compounds 11-26 from scaffold I or II 
Morpholine acetal I or II(1 eq) was dissolved in toluene(10 mL mmol-1), then 2,6-lutidine (2 eq) and Fmoc-aa- $\mathrm{Cl}(1 \mathrm{eq})$ were sequentially added. The reaction mixture was brought to60 $\mathrm{C}$

195 and stirred for $2 \mathrm{~h}$, then allowed to return at r.t. Additional toluene $(5 \mathrm{ml} \mathrm{mmol}-1)$ and $\mathrm{p}$-toluene sulfonic acid monohydrate ( $5 \mathrm{eq}$ ) were added, then the mixture was placed in a single-necked round-bottomed flask equipped with a reflux condenser and a dropping funnel containing $4 \mathrm{~A}$ ${ }^{\circ}$ molecular sieves and it was refluxed for $2 \mathrm{~h}$. The reaction mixture was brought back at r.t. and $30 \%$ diethylamine solution in $\mathrm{CH} 3 \mathrm{CN}(5 \mathrm{~mL} \mathrm{mmol}-1)$ was added. The reaction mixture

200 was stirred for $2 \mathrm{~h}$ at r.t., then diluted with EtOAc ( $40 \mathrm{~mL} \mathrm{mmol}-1)$, washed with $5 \% \mathrm{NaHCO}_{3}$ and brine. The organic layers were dried over anhydrous Na2SO4, filtered and evaporated. A dark brown oil was obtained and purified by flash chromatography (EtOAc-petroleum ether $2: 1$ ), giving the title2,5-diketopiperazine compounds. See ESI file for characterization data.

\section{General procedure $C$ for the synthesis of compounds 27-35 from scaffold I}

205 Morpholine acetal I (1 eq) was dissolved in anhydrous $\mathrm{CH}_{2} \mathrm{Cl}_{2}(4 \mathrm{~mL} \mathrm{mmol}-1)$, then 2,6lutidine (2 eq) and Fmoc-aa-Cl (1 eq) were sequentially added. The reaction mixture was brought to $60^{\circ} \mathrm{C}$ and stirred for $2 \mathrm{~h}$ under a nitrogen atmosphere. The reaction mixture was then diluted with $\mathrm{CH}_{2} \mathrm{Cl}_{2}$ and sequentially washed with $5 \% \mathrm{HCl}, 5 \% \mathrm{NaHCO}_{3}$ and brine. The organic layers were dried overNa2SO4, filtered and evaporated to give a brownish foam. The

210 crude product was then dissolved in toluene $(5 \mathrm{~mL} \mathrm{mmol}-1)$ and $p$-toluene sulfonic acid monohydrate ( $1 \mathrm{eq})$ was added. The reaction mixture was placed in a single-necked roundbottomed flask equipped with a reflux condenser and a dropping funnel containing $4 \mathrm{~A}^{\circ}$ molecular sieves and refluxed for $2 \mathrm{~h}$, then it was allowed to return to r.t. EtOAc was added, and the organic solution was washed with $5 \% \mathrm{NaHCO}_{3}$ and brine. The organic layers were

215 dried over anhydrous $\mathrm{Na}_{2} \mathrm{SO}_{4}$, filtered and evaporated to give a dark brown oil. The crude product was dissolved in $30 \%$ diethylamine in $\mathrm{CH} 3 \mathrm{CN}(5 \mathrm{~mL} \mathrm{mmol}-1)$ and allowed to react for 
$2 \mathrm{~h}$ at r.t. The reaction mixture was then evaporated to obtain a pale brown solid, which was purified by flash chromatography (EtOAc-petroleum ether $3: 1$ ), to give the title bicyclic 2 oxopiperazine compounds as dark orange oils. See ESI file for characterization data.

\section{General procedure $D$ for the synthesis of compounds 36-40 and 42 from scaffold IV}

Bromoacetyl-lactonelV7was dissolved in $\mathrm{MeOH}(1 \mathrm{~mL} \mathrm{mmol}-1)$, then selected primary amine (5 eq) and Et3N (5 eq) were added,and the mixture was left reacting in a microwave synthesizer at $100{ }^{\circ} \mathrm{C}$ for $20 \mathrm{~min}$. The mixture was then diluted with $\mathrm{CH} 2 \mathrm{Cl} 2$ and eluted through Amberlist A-21 Amberlist 15 resins. After solvent evaporation, pure compound was obtained

225 without further chromatographic purification. See ESI file for characterization data.

\section{Yeast strains and media}

The wild-type BY4742 (MATahis3 leu2 lys2 ura3) strain was used. The deletion strains used were BY4742Dsnq2(MATahis3leu2 lys2 ura3 snq2::kanMX), BY4742Dpdr3(MATahis3 leu2lys2 ura3 pdr3::kanMX4), and BY4742Derg6(MATahis3 leu2lys2 ura3 erg6::kanMX4). For

230 growth inhibition tests, yeast cells were inoculated at $1 ¥ 105$ cell/ml in YPD medium (Yeast Peptone Dextrose, yeast extract 1\%(w/v), peptone 2\%(w/v) supplemented with $2 \%$ glucose). BY4742FOX3-GFP(MATa his3 leu2 met15ura3 YIL160c-GFP), a strain bearing the fusion product GFP-FOX3, 3-ketoacyl-CoA thiolase, having peroxisomal localization)was used to follow peroxisomal proliferation. For mitochondrial membrane activation and peroxisomal

235 proliferation assays YP added with various Carbon sources was used: 2\% Glucsose (YPD),2\% Galactose (YPGal), 2\% Glycerol (YPGly) and 0.2\% Oleate (YPO, supplemented with $0.2 \%$ Tween 80 to emulsify oleate).

\section{Biological assays}

BY4742 strain was grown in YPD. The cultures was dispensed at equal volume onto 96-well 240 plates, each well containing a different compound at a concentration of $0.3 \mathrm{mM}$. The assay 
plates were incubated at $28^{\circ} \mathrm{C}$ with shaking, and yeast growth was scored by measurement of O.D.650with a Victor high-throughput microplate spectrophotometer equipped with a 96-well plate reader every $2 \mathrm{~h}$ for the first $24 \mathrm{~h}$, and then once a day for 4 days. Compounds responsible for the variation in cell generation time (the time interval required for a yeast cell

245 to divide), or of the O.D.650value of the stationary phase (O.D.st), or both, were selected for further characterization. Cell generation time (GenT, the time interval required for a yeast cell to divide) was calculated as $\mathrm{D}(\mathrm{t} 2-\mathrm{t} 1) /\left\{3.3^{\star}[\log 10(\mathrm{O} . \mathrm{D} . \mathrm{t} 2 / \mathrm{O} . \mathrm{D} . \mathrm{t} 1)]\right\}$, where $\mathrm{t} 1$ and $\mathrm{t} 2$ are time points flanking the inflection point of the growth curves. The percent effect of each molecule was calculated as a function of the GenT or O.D.st value of treated cells and the GenT or

250 O.D.st of untreated cells. The effects on deletant stains was assayed for selected compounds.

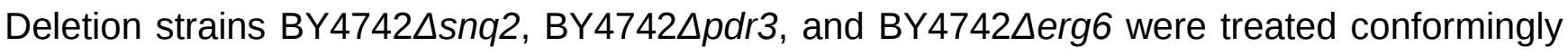
with the wild-type strain treatment, with $0.3 \mathrm{mM}$ molecules. Compounds responsible for the variation in deletant strain cells generation time, or of the O.D.st value, or both, were selected for mitochondrial and peroxisomal investigations.

\section{Fluorescence microscopy}

Effects at the mitochondrial level of selected compounds were observed by mitochondrial membrane potential investigation. Effects at the peroxisomal level were investigated by GFPFOX3microscopic observation. After $4 \mathrm{~h}$ of treatment with selected compounds at $0.3 \mathrm{mM}$ concentration, or without them as a control, cultured cells were dissolved at $1 ¥ 106$ cells ml-1in

26010 mM HEPES buffer, pH 7.4, containing 5\% glucose. Rhodamine B hexylester was added to a final concentration of $100 \mathrm{nM}$. After 15-30 min of incubation, the mitochondrial membrane potential was visualized by fluorescence microscopy (excitation at $555 \mathrm{~nm}$,green/emission at $579 \mathrm{~nm}, \mathrm{red}$ ). Each aliquot was treated with Calcofluor White (M2R) (Blue) to evidence the cell wall in orderto count total cells. Mitochondrial membrane potential activation was 
265 calculated as percentage of red-fluorescent cells (rhodamine B hexyl ester-labelled cells) toward total blue-cells (Calcofluor White-labelled cells) of the same treated culture.

\section{Acknowledgements}

Fondazione Roma, CINMPIS, and SYBARIS (Grant Agreement 242220) are acknowledged for financial support. Dr Cristina Faggiis gratefully acknowledged for the X-ray crystallographic 270 analyses.

\section{Notes and references}

${ }^{1}$ B. R. Stockwell, Nature, 2004,432, 846.

2(a) L. M. Mayr and D. Boanic, Curr. Opin. Pharmacol., 2009,9, 580-588; (b) I. Stefanini, A. Trabocchi, E. Marchi, A. Guarna and D. Cavalieri, J. Biol. Chem., 2010,285, 23477.

275 33(a) S. Hoon, R. P. St Onge, G. Giaever and C. Nislow,TrendsPharmacol. Sci., 2008,29, 499;

(b) M. A. Resnick and B. S. Cox, Mutat Res., Fundam. Mol. Mech. Mutagen., 2000,451,1; (c) C. Brenner,GenomeBiology, 2004,5, 240; (d) D. Auerbach, A. Arnoldo, B. Bogdan,M. Fetchko and I. Stagljar,Curr. Proteomics, 2005,2,1.

${ }^{4}$ (a) G. Giaever, P. Flaerty, J. Kumm, M. Proctor, C. Nislow, D. F.Jarmillo, A. M. Chu, M. I. 280 Jordan, A. P. Arkin and R. W. Davis, Proc. Natl. Acad. Sci. U. S. A., 2004,101, 793; (b) P. Y. Lum, C. D. Armour,S. B. Stepaniants, G. Cavet, M. K. Wolf, J. S. Butler, J. C. Hinshaw, P. Garnier, G. D. Prestwich, A. Leonardson, P. Garrett-Engele, C. M.Rush, M. Bard, G. Schimmack, J. W. Phillips, C. J. Roberts and D. D.Shoemaker, Cell, 2004,116, 121; (c) A.B.Parsons, R.L.Brost, H.Ding, Z. Li, C. Zhang, B. Sheikh, G. W. Brown, P. M. Kane, T. 285 R.Hughes and C. Boone,Nat. Biotechnol., 2004,22, 62; (d) A. B. Parsons,A. Lopez, I. E. Givoni, D. E. Williams, C. A. Gray, J. Porter, G. Chua,R. Sopko, R. L. Brost, C. H. Ho, J. Wang, T. Ketela, C. Brenner, J. A.Brill, G. E. Fernandez, T. C. Lorenz, G. S. Payne, S. 
Ishihara, Y. Ohya,B. Andrews, T. R. Hughes, B. J Frey, T. R. Graham, R. J. Andersen andC. Boone,Cell, 2006,126, 611.

$290{ }^{5}$ J. I. Levin, J. M. Chen, L. M. Laakso, M. Du, X. Du, A. M. Venkatesan,V. Sandanayaka, A. Zask, J. Xu, W. Xu, Y. Zhang and J. S. Skotnicki,Bioorg. Med. Chem. Lett., 2005,15, 4345.

${ }^{6}$ N. G. Almstead, R. S. Bradley, S. Pikul, B. De, M. G. Natchus, Y. O.Taiwo, F. Gu, L. E. Williams, B. A. Hynd, M. J. Janusz, C. M. Dunawayand G. E. Mieling,J. Med. Chem., 1999,42, 4547.

$295{ }^{7}$ C. Lalli, A. Trabocchi, F. Sladojevich, G. Menchi and A. Guarna,Chem.-Eur. J., 2009,15, 7871.

${ }^{8}$ A. Trabocchi, F. Sladojevich and A. Guarna,Chirality, 2009,21, 584.

${ }^{9}$ (a) M.B.Martinsandl.Carvalho, Tetrahedron, 2007,63, 9923; (b)B.Nicholson, G. K. Lloyd, B. R. Miller, M. A. Palladino, Y. Kisob, Y.Hayashib and S. T. C. Neuteboom,Anti-Cancer Drugs, 300 2006,17, 25;(c) A. Folkes, M. B. Roe, S. Sohal, J. Golec, R. Faint, T. Brooks andP. Charlton,Bioorg. Med. Chem. Lett., 2001,11, 2589; (d) S. Wang, J.Golec, W. Miller, S. Milutinovic, A. Folkes, S. Williams, T. Brooks, K.Hardman, P. Charlton, S. Wren and J. Spencer,Bioorg. Med. Chem.Lett., 2002,12, 2367; (e) A. P. Einholm, K. E. Pedersen, T. Wind, P.Kulig, M. T. Overgaard, J. K. Jensen, J. S. BØdker, A. Christensen, P. Charlton and P. A. 305 Andreasen, Biochem. J., 2003,373, 723; (f)D.R.Houston, B. Synstad, V. G. H. Eijsink, M. J. R. Stark, I. M. Egglestonand D. M. F. Van Aalten,J. Med. Chem., 2004,47, 5713; (g) F. Fdhila,V. V'azquez, J. L. S'anchez and R. Riguera,J. Nat. Prod., 2003,66,1299-1301; (h) M. Teixid, E. Zurita, M. Malakoutikhah, T. Tarrag and E. Giralt, J. Am. Chem. Soc., 2007,129, 11802.

${ }^{10}$ F. Sladojevich, A. Trabocchi and A. Guarna, Org. Biomol. Chem., 2008,6, 3328.

$310^{11}$ P. Reungpatthanaphong, S. Dechsupa, J. Meesungnoen, C. Loetchuti-nat and S. Mankhetkorn, J. Biochem. Biophys. Methods, 2003,57,1. 
${ }^{12}$ H. Van Den Bosch, R. B. Schutgens, R. J. Wanders and J. M. Tager, Annu. Rev. Biochem., 1992,61, 157.

13 B. E. Bauer, H. Wolfger and K. Kuchler, Biochim. Biophys. Acta, Biomembr., 1999,1461, 315217. 


\section{Schemes}

Scheme 1 Generation of morpholine scaffolds through three different pathways.

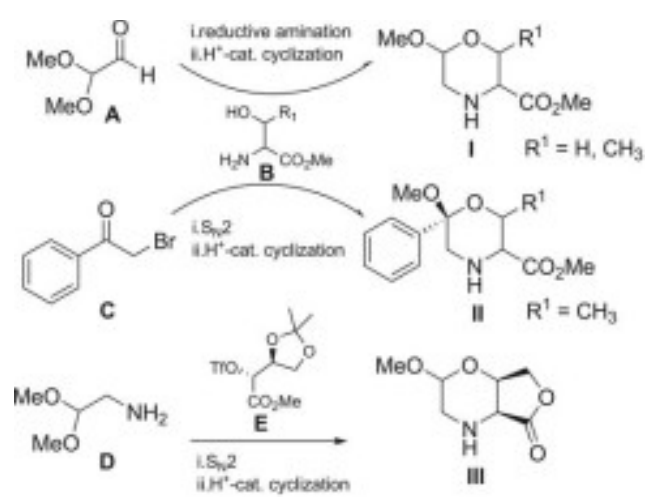


Scheme 2 Library members from scaffolds I-II.

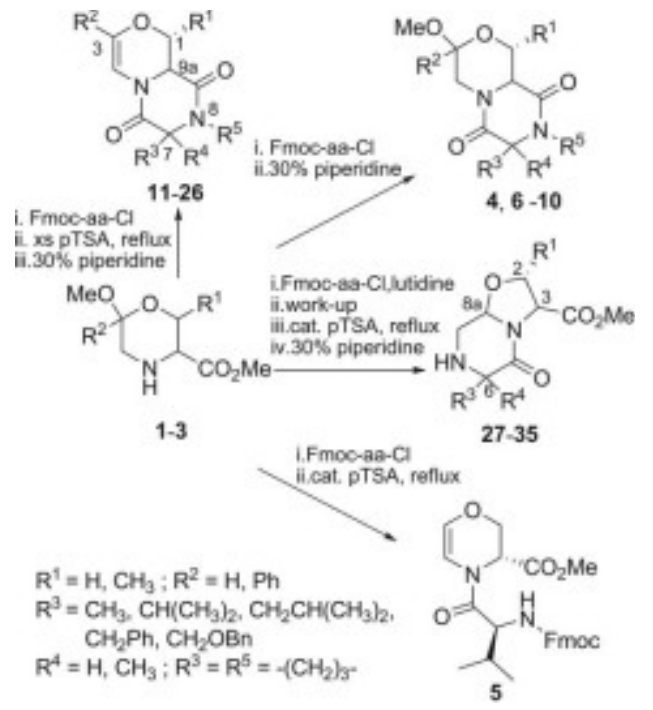


Scheme 3 Library members from scaffold III.

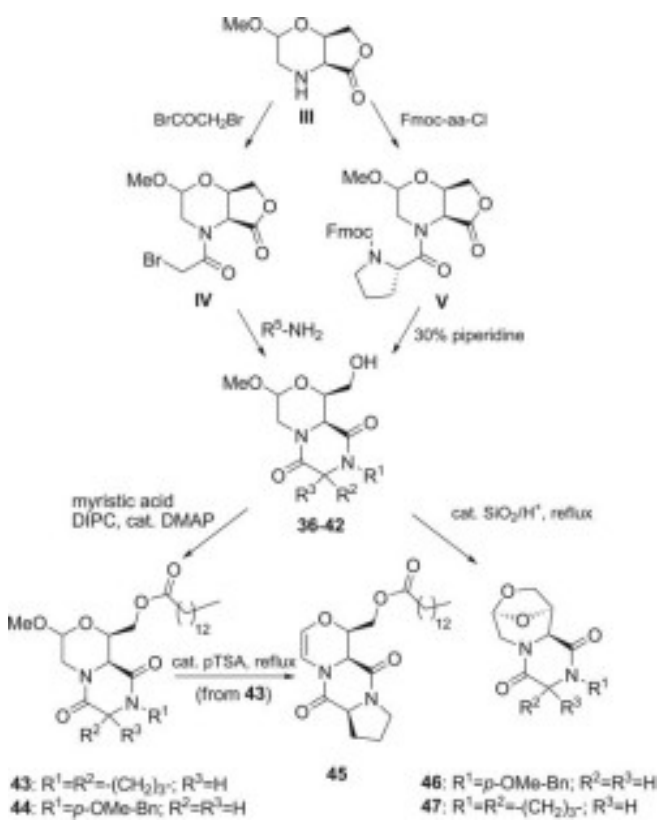




\section{Figures}

Fig. 1 X-Ray structure of 34 .

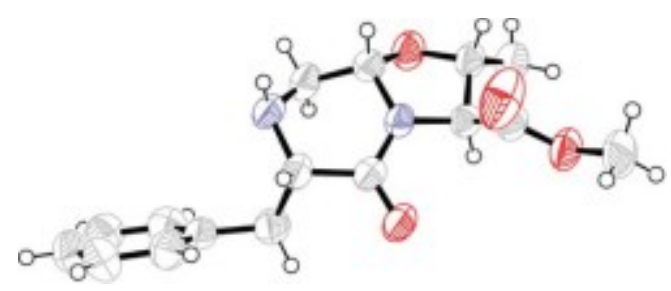


Fig. 2 Graphical representation of the effects of the molecules a) on the BY4742 wild-type strain b) on the BY4742Derg6 strain c) on the BY4742Dsnq2 strain and d) on the BY4742Dpdr3 strain. Percent values on both axes are referred to the untreated strain.

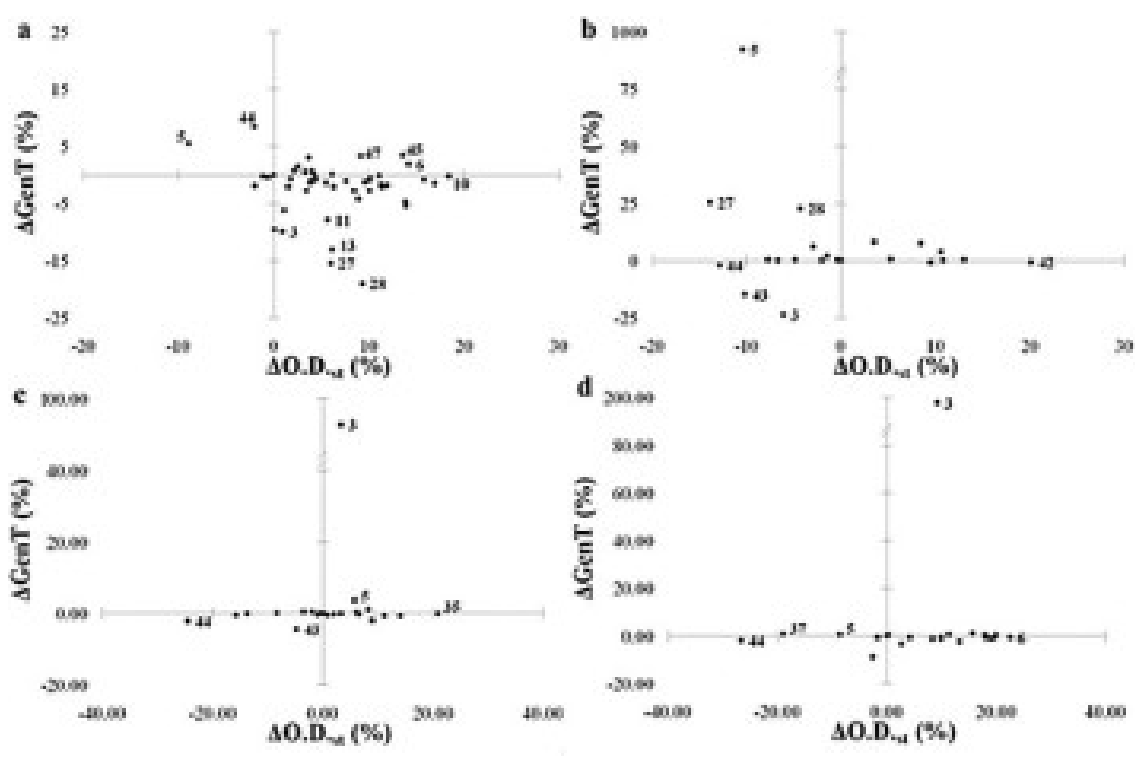


325 Fig. 3 Mitochondrial activation induced by the selected molecules on theBY4742 strain grown in various media. Values indicate the mean of three measurements, error bars indicate the corresponding standard deviations values. Student's t test was used to evaluate the data significance, * $p<0.05 ;{ }^{*}, p<0.01$.

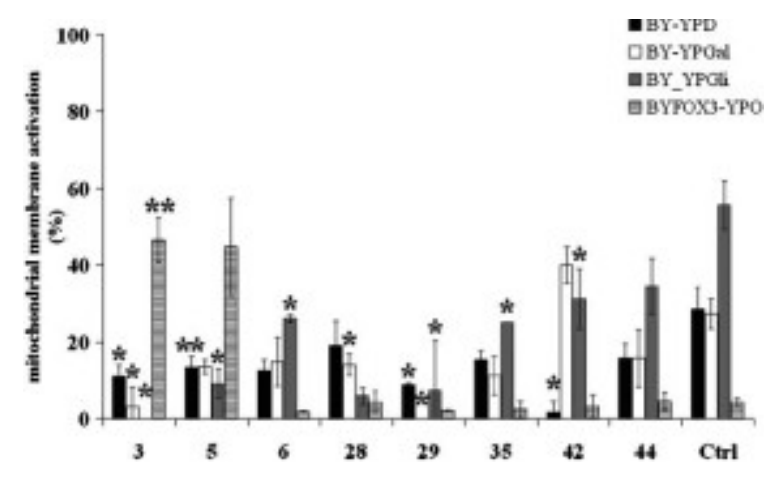


Fig. 4 Peroxisomal proliferation induced by the treatment of the strainBY4742-GFP/FOX3 with 330 the selected molecules. Values indicate the mean of tree measurements, error bars indicate the corresponding standard deviations values. Student's $t$ test was used to evaluate the data significance, ${ }^{*}, p<0.05 ;{ }^{* *}, p<0.01$

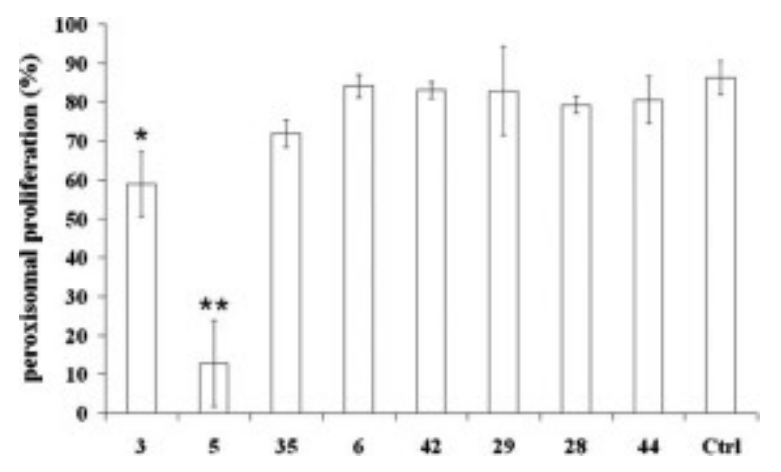


Fig. 5 Microscopic observation of the effects of eight selected molecules on the BY4742FOX3-GFP strain grown in YPO (0.2\%). a) cells wall stained with Calcofluor White b) 335 mitochondrial membrane potential activation visualized with Rhodamine B hexyl ester c) peroxisomes individuated by fusion product FOX3-GFP expression, a 3-ketoacyl-CoAthiolase, having peroxisomal localization.

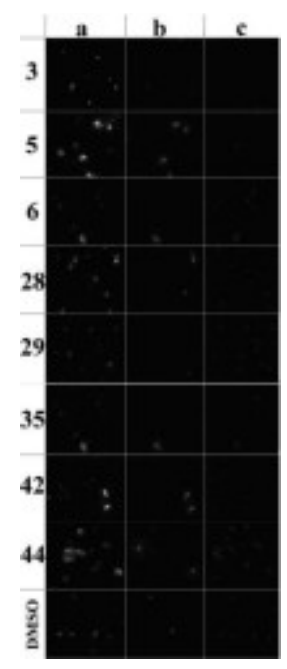

